\section{References}

1. Tanishima K, Hayashi T, Mochikawa $Y$, et al. Specific inhibition of the activity of lactate dehydrogenase isoenzyme composed of $\mathbf{M}$ subunit by 1,6-hexanediol. Seikaq (Jpn) 1984;56:638.

2. Tanishima $K$, Hayashi $T$, Matsushima $M$, et al. Activity of lactate dehydrogenase isoenzymes $L D_{1}$ and $L D_{2}$ in serum as determined by using an inhibitor of the M-subunit. Clin Chem 1985;31:1175-7.

3. Usategui-Gomez M, Wicks RW, Warshaw M. Immunochemical determination of the heart isoenzyme of lactate dehydrogenase $\left(\mathrm{LDH}_{1}\right)$ in human serum. Clin Chem 1979;25:729-34.

4. Wolf RE, Graeber GM, Burge JR, et al. Evaluation of serum creatine kinase and lactate dehydrogenase in experimental myocardial infarction, atriotomies and thoracotomies. Ann Thorac Surg 1986;41:378-86.

5. Graeber GM, Shawl FA, Head HD, et al. Changes in serum creatinine kinase and lactate dehydrogenase caused by acute perioperative myocardial infarction and by transatrial candiac surgical procedures. J Thorac Cardiovasc Surg 1986;92:63-72.

\title{
Lactate and Pyruvate Electrochemical Biosensors for Whole Blood in Extracorporeal Experiments with an Endocrine Artificial Pancreas
}

Marco Mascini, Franco Marcel, ${ }^{1}$ Danila Moscone, Gluseppe Calabrese, ${ }^{2}$ and Maseimo Masel Benedottr

Glucose, lactate, and pyruvate can be continuously measured in whole blood by using three extracorporeal electrochemical biosensors. We fixed two newly developed sensors, for lactate and pyruvate, in series with an endocrine artificial pancreas ("Betalike") without affecting its performance. These new sensors for use with whole blood should be able to clarify the fate of the intermediary metabolites of glucose in diabetic patients receiving infusions of drugs or insulin.

Additional Keyphrases: diabetes - insulin infusion - metabolism - glucose sensor - immobilized enzymes

The principal aim in therapy of diabetes mellitus is to correct metabolic alterations caused by the lack of insulin. Recently, automatic systems have been introduced for delivering insulin, with feedback mechanisms that are regulated by blood-glucose concentrations. Although these instruments represent a great improvement in therapy, they still do not completely normalize the altered concentrations of intermediary metabolites, in part because peripheral blood glucose is unsuitable as the only signal on which to base the control algorithms of the artificial pancreas. Once adequate systems for continuous monitoring of metabolites have been realized, algorithms based on multiple metabolic signals presumably will be developed (1).

Here we report the development of a pyruvate sensor, which we coupled with an L-lactate sensor recently reported (2-4) and placed downstream from a newly developed artificial pancreas, the "Betalike" (Ansaldo Div., Elettronica Esacontrol S.p.A., Genova, Italy). The Betalike dialyzes the blood diluted with a pH 7.40 physiological solution (sodium chloride $5.26 \mathrm{~g} / \mathrm{L}$, sodium acetate $2.22 \mathrm{~g} / \mathrm{L}$, sodium gluconate $5.02 \mathrm{~g} / \mathrm{L}$, potassium chloride $0.37 \mathrm{~g} / \mathrm{L}$, and magnesium chloride $0.14 \mathrm{~g} / \mathrm{L}$, supplied by Abbott S.p.A., Aprilia 04100 , Italy), re-infuses the blood cells into the patient's bloodstream, and analyzes the dialysate for glucose.

Istituto Chimica Analitica Università di Firenze, 50121 Firenze, Italy.

1 Dip. Scienze e Tecnologie Chimiche, II Univ. Roma Tor Vergata, 00173 Roma, Italy.

2 Istituto di Patologia Speciale Medica, Università di Perugia, Perugia, Italy.

Received September 15, 1986; accepted January 12, 1987.
Adding the new sensors to the Betalike permitted monitoring of glucose, lactate, and pyruvate in real time, in a protein-free dialysate with viscosity similar to that of an aqueous solution.

The pyruvate sensor is based on commercially available pyruvate oxidase (EC 1.2.3.3; from Pediococcus 8p.), which is immobilized on the surface of an hydrogen peroxide electrochemical sensor (4). The lactate sensor is based on lactate oxidase (EC 1.1.3.2; from Pediococcus sp.) immobilized on an oxygen sensor.

\section{Materlals and Methods}

\section{Materials}

The L-lactate oxidase (activity $18 \mathrm{kU} / \mathrm{g}$ ) and pyruvate oxidase (activity $21 \mathrm{kU} / \mathrm{g}$ ) were obtained from Toyo Yozo Co., Shizuoka, Japan. Cellulose acetate (53\% acetyl) and high- $M_{\mathrm{r}}$ poly(vinyl acetate) were from Farmitalia Carlo Erba, Milano, Italy. Biodyne Immunoaffinity Membrane, nylon 6-6 (adipic acid-hexamethylenediamine), porosity 0.2 $\mu \mathrm{m}$, with carboxylic groups on the surface, was from Pall Corp., Glen Cove, NY 11542. Dialysis membrane, 0.001 inch $(25 \mu \mathrm{m})$ in thickness, $M_{\mathrm{r}}$ cutoff 12000 , was from A. H. Thomas Co., Philadelphia, PA 19105. A hydrogen peroxide sensor and a flow-through cell with a dead space of $40 \mu \mathrm{L}$ were from Instrumentation Laboratory S.p.A., Milano, Italy. We used platinum wire $0.5 \mathrm{~mm}$ in diameter and a silverfoil cathode with $0.5 \mathrm{~cm}^{2}$ surface area. The polarization unit was an electrochemical detector used with liquid chromatography (Model VA 641; Metrohm, Herisau, Switzerland). The current was measured through a recorder (Omniscribe, Houston Instruments, Austin, TX 78761). In casting the cellulose membrane we used a precision gauge tool (Precision Gage and Tool Co., Dayton, OH 45401).

\section{Procedures}

Lactate sensor. The procedure for assembling the lactate sensor has been described and discussed elsewhere $(3,4)$.

Pyruvate sensor. The pyruvate sensor was a hydrogen peroxide sensor ( $\mathrm{Pt}$ vs $\mathrm{Ag}-\mathrm{AgCl}$ ) covered with a membrane containing immobilized pyruvate oxidase. The sensor was protected with a cellulose acetate membrane.

Casting the cellulose acetate membrane. To protect the anode from interfering (potentially oxidizable) compounds, 
we prepared cellulose acetate membrane by mixing $40 \mathrm{~mL}$ of cyclohexanone, $60 \mathrm{~mL}$ of acetone, $3.96 \mathrm{~g}$ of cellulose acetate, and $40 \mathrm{mg}$ of high- $M_{\mathrm{r}}$ poly(vinyl acetate). The solution was cast on a glass plate to give a thickness of $\mathbf{2 0 0}$ $\mu \mathrm{m}$. After evaporating excess solvent at room temperature for 30-40 min, we immersed the glass plates and membranes in water, peeled off the membranes, dried them with abeorbent paper, and stored them at room temperature just as for the usual dialysis membrane. The dried membrane is $20-\mu \mathrm{m}$ thick.

Immobilization of pyruvate oxidase. A newly developed commercially available nylon $6-6$ filter membrane, $0.8 \mathrm{~cm}$ in diameter, with carboxylic groups on the surface, was soaked with $0.1 \mathrm{~mol} / \mathrm{L}$ solution of 1-ethyl-3-(3-dimethylaminopropyl)carbodiimide in phosphate buffer $(0.5 \mathrm{~mol} / \mathrm{L}, \mathrm{pH} 4.8)$ for $40 \mathrm{~min}$ at room temperature, with continuous stirring. The membrane was then washed with a bicarbonate buffer $(0.4$ $\mathrm{mol} / \mathrm{L}, \mathrm{pH} \mathrm{7.0)}$, and we mixed on its surface 1-2 $\mathrm{mg}$ of enzyme in $10 \mu \mathrm{L}$ of the bicarbonate buffer, and left this in a humid atmosphere at $4^{\circ} \mathrm{C}$ overnight.

Assembly of the sensor. We first wrapped the surface of the platinum wire with a membrane of cellulose acetate to eliminate interferences from electroactive chemicals such as ascorbic acid. A second membrane with immobilized enzyme was placed on top of the first. The third (outer) membrane was a dialysis membrane, which prevented microbial degradation of the enzyme and kept the enzyme from being leached out of the second membrane. The three layers were held together with a rubber 0-ring. When not in use the sensor was kept in a $1 \mathrm{mmol} / \mathrm{L}$ solution of FAD in Tris buffer, pH 8.0.

\section{Results and Discussion}

\section{Assay Validation}

The analytical behavior of lactate and pyruvate sensors has been previously reported in detail $(2-4)$. The lactate sensor was used previously for in vivo experiments, and such results will not be reported here. The reaction of pyruvate in the presence of pyruvate oxidase is as follows:

$$
\text { Pyruvate }+\mathrm{HPO}_{4}^{2-}+\mathrm{O}_{2}+\mathrm{H}_{2} \mathrm{O} \rightarrow \text { acetyl phosphate }+
$$$$
\mathrm{H}_{2} \mathrm{O}_{2}+\mathrm{CO}_{2}
$$

Therefore a sensor for oxygen or hydrogen peroxide could be used to monitor the reaction, but a carbon dioxide sensor would be unsuitable because of the low concentration of pyruvate and the high bicarbonate content of blood.

For clinical determination of pyruvate a hydrogen peroxide sensor must be used, because the normal range of pyruvate in blood (40-120 $\mu \mathrm{mol} / \mathrm{L})$ is too low to be determined with an oxygen sensor. (Recall that the lactate sensor is realized via an oxygen sensor because of the higher normal range for lactate in blood.) The reaction proceeds in the presence of several cofactors that optimize the reaction. Detailed behavior of such cofactors is reported elsewhere (5), as are critical values as evaluated in in vitro experiments. From these experiments we concluded that the optimum concentrations of cofactors in the samples for pyruvate determination should be $0.1 \mathrm{mmol}$ of thiamin pyrophosphate, $0.5 \mathrm{mmol}$ of phosphate, and $2.5 \mathrm{mmol}$ of calcium chloride per liter of Tris buffer ( $\mathrm{pH} 7.0,40 \mathrm{mmol} / \mathrm{L})$.

Table 1 reports the pyruvate concentrations in serum from normal and diabetic patients, as determined spectrophotometrically and with the pyruvate sensor procedure. The spectrophotometric analyses involved measuring

\begin{tabular}{cc}
\hline Table 1. Pyruvate Concentrations in Elght Serum \\
Samples as Determined with the Amperometric \\
Pyruvate Sensor and Spectrophotometrically \\
Amperometric sensor & 0.35 \\
0.34 & 0.27 \\
0.28 & 0.28 \\
0.26 & 0.28 \\
0.30 & 0.30 \\
0.29 & 0.29 \\
0.31 & 0.10 \\
0.12 & 0.16 \\
0.15 & \\
Results are mean of three determinations. & \\
\hline
\end{tabular}

$\mathrm{NADH}$ absorbance at $340 \mathrm{~nm}$ in samples treated with lactate dehydrogenase (EC 1.1.1.27) (6). The assay with the pyruvate sensor involved mixing $0.5 \mathrm{~mL}$ of serum with $5 \mathrm{~mL}$ of Tris buffer containing all the cofactors specified above. Results by the two methods correlated well: $r=0.975$ ( $y=$ $1.02 x-0.006 \mathrm{mmol} / \mathrm{L})$.

The pyruvate sensor shows a stable response for at least 30 days, after an initial decrease of about $15 \%$ in the first two days. This variation, which is apparently a constant feature of enzyme sensors, is attributable to enzyme molecules that are weakly linked to the polymeric surface and leach out within $48 \mathrm{~h}$.

\section{Experiments with the Betalike Artificial Pancreas}

The flow cell containing lactate and pyruvate sensors was placed downstream from the glucose sensor of the Betalike apparatus. Dialysate flow rate was fixed at $0.5 \mathrm{~mL} / \mathrm{min}$. Through a T-connection, a peristaltic pump introduced into the flowing dialysate a concentrated solution of cofactors at a flow rate of $0.05 \mathrm{~mL} / \mathrm{min}$, to provide the optimum concentrations of cofactors in the solution entering the flow cell.

Figure 1 shows the results of an "in vivo" determination of glucose, L-lactate, and pyruvate in heparinized blood from a normal subject (a volunteer, nondiabetic, 23-year-old man) being treated with the artificial pancreas (Betalike).

Blood taken from the subject via a double-lumen catheter $(6 \mathrm{~mL} / \mathrm{h})$ was heparinized, diluted 10-fold with physiological buffer, then dialyzed through the dialysis filter. The dialysate was pumped $(0.5 \mathrm{~mL} / \mathrm{min})$ through the glucose sensor, then through the flow cell containing the lactate and pyruvate sensors.

Every 15-20 min we sampled blood from this subject and assayed lactate and pyruvate spectrophotometrically within the next few days. These results, represented as dots in Figure 1, illustrate the comparability of results by the two methods. The good correlation between results by continuous monitoring and by spectrophotometry excludes the possibility that there is reciprocal interference between different sensors. The possibility that the oxygen consumed at the glucose sensor might interfere with the performance of the lactate or pyruvate sensors was excluded in preliminary experiments in which standard solutions of lactate and pyruvate, with or without glucose, gave the same signals. Moreover, one feature of an enzyme sensor is that the enzymatic reaction occurs only at the electrode surface in a very limited area, the bulk concentration of the sample being unaffected by such measurement. This limited extent of the reaction means that the sample composition is not notably altered by the act of measurement. Such is the case here. The consumption of oxygen, calculated from the nanoamperes measured at the glucose electrode, shows a possible 


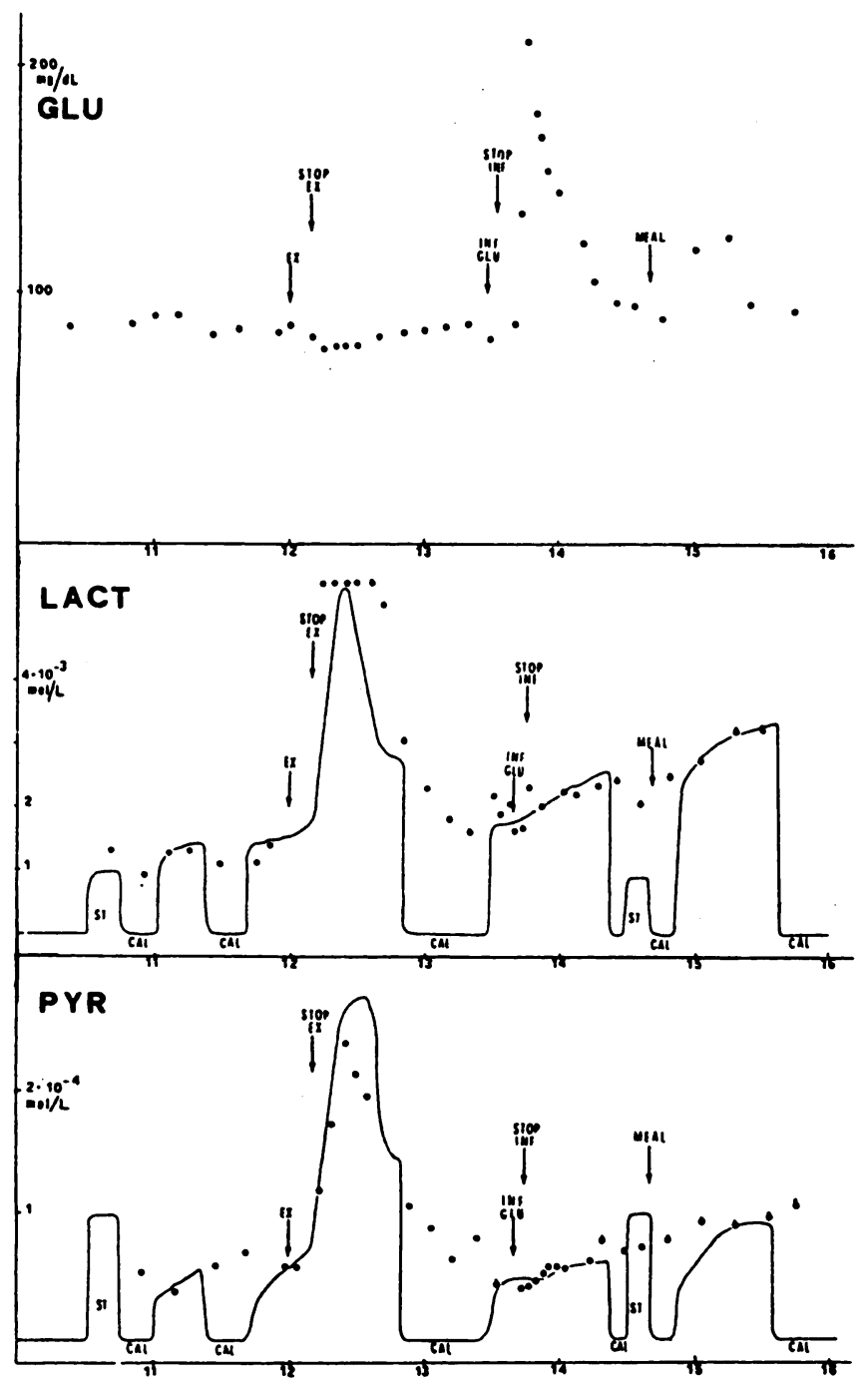

Fig. 1. Continuous monitoring of glucose, lactate, and pynuate in vivo during an experiment with the artificial pancreas, "Betalike" See text for explanations of abbreviations and procedure

decrease of oxygen of only a few nanomoles per liter, i.e., very negligible.

As Figure 1 illustrates, at the time marked ST, a standard solution of lactate and pyruvate was passed through the cell to calibrate the sensors. At the time marked CAL, the glucose sensor of Betalike was calibrated. The blood flow was disconnected from the sensors during such periods. At the time marked EX, the patient was requested to do a short physical exercise, which was stopped at the time marked STOPEX. At the time INFGLU, a 50-g load of glucose was rapidly infused. MEAL indicates the time at which the patient ate a normal meal.

It is interesting to note the large effect of physical exercise on the concentration of lactate and, even more, on pyruvate, followed by the parallel return of both analytes to "normal" concentrations. As expected, the glucose load had a large effect on the glucose concentration, but caused only small variations in the concentrations of lactate and pyruvate. After the meal, all three analytes showed large and continuous variations.

Numerous factors influence fuel consumption; each can produce different variations in metabolic substrates. The lack of insulin that characterizes diabetes causes these variations to assume pathological values.

Correction of these variations to complete normalization will be possible only if the insulin-replacement therapy is regulated on the basis of the complex relationships among the intermediate metabolites (2).

The opportunity to create algorithms that induce optimal therapeutic results is strictly related to the amount of information available. The reliability of the immobilization procedures described here provides diabetological studies with a new possibility for regulating insulin therapy, as well as interesting speculations regarding a "cure" for diabetes.

We are grateful to Toyo Yozo Co. for the L-pyruvate oxidase; Prof. P. Brunetti for valuable discussion; Instrumentation Laboratory S.p.A., Milano, for providing the hydrogen peroxide flow cell; and the Consiglio Nazionale delle Ricerche, Progetto Chimica Fine Secondaria, for financial support.

\section{References}

1. Fogt EJ, Dodd LM, Clemens AH. Development and evaluation of a glucose analyzer for a glucose-controlled insulin infusion system (Biostator). Clin Chem 1978;24:1366-72.

2. Second Assisi International Symposium on Advanced Models for the Therapy of Insulin Dependent Therapy, Assisi, Italy, 20-23 April, 1986, Sesesion IV.

3. Mascini M, Fortunati S, Moscone D, Palleschi G, Massi-Benodetti M, Fabietti P. An L-lactate sensor with immobilized entyme for use in vivo studies with an endocrine artificial pancreas. Clin Chem 1985;31:451-3.

4. Mascini M, Moscone D, Pallechi G. A L-lactate electrode with L lactate oxidase immobilized on nylon net for blood serum sample in flow system. Anal Chim Acta 1984;157:45-51.

5. Mascini M, Mazmei F. Amperometric sensor for pyruvate with immobilized pyruvate oxidase. Anal Chim Acta, in prees.

6. Sigma Chemical Co., St. Louis, MO 63178. Technical Bulletin No. 726-UV. 